\title{
GENERALIZED DERIVATIONS IN PRIME RINGS AND NONCOMMUTATIVE BANACH ALGEBRAS
}

\author{
VinCEnZo De Filippis
}

\begin{abstract}
Let $R$ be a prime ring of characteristic different from $2, C$ the extended centroid of $R$, and $\delta$ a generalized derivations of $R$. If $[[\delta(x), x], \delta(x)]=0$ for all $x \in R$ then either $R$ is commutative or $\delta(x)=$ ax for all $x \in R$ and some $a \in C$. We also obtain some related result in case $R$ is a Banach algebra and $\delta$ is either continuous or spectrally bounded.
\end{abstract}

\section{Introduction}

Let $R$ be an algebra with center $Z(R)$ and radical Jacobson $\operatorname{rad}(R)$. Let $d: R \rightarrow R$ be a linear operator on $R$ such that $d(x y)=d(x) y+x d(y)$ for all $x, y \in R$. In literature such a mapping is called (linear) derivation of $R$. In particular $d$ is an inner derivation, induced by an element $q \in R$, if $d(x)=[q, x]$ for all $x \in R$.

The classical result of Singer and Werner in [21] says that any continuous derivation on a commutative Banach algebra has the range in the Jacobson radical of the algebra. Singer and Werner also formulated the conjecture that the continuity assumption can be removed. In 1988 Thomas verified this conjecture in $[22]$.

Of course the same result of Singer and Werner does not hold in noncommutative Banach algebras (because of inner derivations). Hence in this context a very interesting question is how to obtain noncommutative version of SingerWerner theorem. A first answer to this problem has been obtained by Sinclair in [20]. He proved that every continuous derivation of a Banach algebra leaves primitive ideals of the algebra invariant. Since then many authors obtained more information about derivations satisfying certain suitable conditions in Banach algebras.

In [23] Vukman proved that if $d$ is a linear derivation of a noncommutative semisimple Banach algebra $R$ such that $[d(x), x] d(x)=0$ for all $x \in R$, then $d=0$.

Received April 11, 2007.

2000 Mathematics Subject Classification. Primary 16N60; Secondary 16W25.

Key words and phrases. prime ring, derivations, differential identities, Banach algebras. 
In [16] Mathieu and Murphy proved the result that if $d$ is a continuous derivation on an arbitrary Banach algebra such that $[d(x), x] \in Z(R)$ for all $x \in R$, then $d$ maps into the radical. Later in [17] Mathieu and Runde removed the continuity assumption using the classical result of Posner on centralizing derivations of prime rings in [19] and Thomas' theorem in [22]: they showed that if $d$ is a derivation which satisfies $[d(x), x] \in Z(R)$ for all $x$ in a Banach algebra $R$, then $d$ has its range in the radical of the algebra.

More recently in [18] Park proved that if $d$ is a linear continuous derivation of a noncommutative Banach algebra $R$ such that $[[d(x), x], d(x)] \in \operatorname{rad}(R)$ for all $x \in R$, then $d(R) \subseteq \operatorname{rad}(R)$.

Here we will continue the investigation about the relationship between the structure of an algebra $R$ and the behavior of derivations defined on $R$.

Our first aim is to give a generalization of the Park's result for prime rings, by replacing the derivation $d$ by a special additive mapping $\delta$ of $R$ defined as follows: for all $x, y \in R, \delta(x y)=\delta(x) y+x d(y)$ for some derivation $d$ of $R$. Such a mapping $\delta$ is called generalized derivation of $R$. The simplest example of generalized derivation is a map of the form $g(x)=a x+x b$, for some $a, b \in R$ : such generalized derivations are called inner. Generalized inner derivations have been primarily studied on operator algebras. Therefore any investigation from the algebraic point of view might be interesting (see for example [7], [13]). In this sense, our first goal is to prove the following result for prime rings:

Theorem 1. Let $R$ be a prime ring of characteristic different from $2, C$ the extended centroid of $R$, and $\delta$ a generalized derivations of $R$. If $[[\delta(x), x], \delta(x)]=0$ for all $x \in R$, then either $R$ is commutative or $\delta(x)=$ ax for all $x \in R$ and some $a \in C$.

Then we apply this result to the study of analogous conditions for continuous generalized derivations on Banach algebras. More precisely we will prove:

Theorem 2. Let $R$ be a non-commutative Banach algebra, $\delta=L_{a}+\delta a$ continuous generalized derivations of $R$, where $L_{a}$ denote the left multiplication by some element $a \in R$ and $d$ is a derivation of $R$. If $[[\delta(x), x], \delta(x)] \in \operatorname{rad}(R)$ for all $x \in R$, then $[a, R] \subseteq \operatorname{rad}(R)$ and $d(R) \subseteq \operatorname{rad}(R)$.

\section{The matrix case}

We begin with the case when $R$ is a ring of matrices over a field and $\delta$ is an inner generalized derivation. We have:

Lemma 1. Let $R=M_{k}(F)$ be the ring of $k \times k$ matrices over the field $F$ of characteristic different from 2, with $k>1$, a,b elements of $R$ such that $[[a x+x b, x], a x+x b]=0$ for all $x \in R$. Then $a, b \in Z(R)$.

Proof. Let $\left\{e_{i j}\right\}$ be the standard basis for $R$ and $I$ the identity matrix of $R$. The first aim is to prove that $b-a$ is a central matrix. Denote $a=\sum a_{r s} e_{r s}$, 
$b=\sum b_{r s} e_{r s}$ for some $a_{r s}, b_{r s} \in F$. Let $x=e_{i j}$ for any $i \neq j$. We have

$$
0=[[a x+x b, x], a x+x b]=\left[\left[a e_{i j}+e_{i j} b, e_{i j}\right], a e_{i j}+e_{i j} b\right]=-e_{i j}\left(b_{j i}-a_{j i}\right)^{2} .
$$

Thus $b_{j i}=a_{j i}$ for all $j \neq i$, that is, $(b-a)$ is a diagonal matrix. For any $\psi \in \operatorname{Aut}_{F}(R)$, we have $[[\psi(a) x+x \psi(b), x], \psi(a) x+x \psi(b)]=0$ and so, by the previous case, $\psi(b-a)$ is a diagonal matrix. In particular, for any $r \neq s$, if $\psi(x)=\left(I+e_{r s}\right) x\left(I-e_{r s}\right)$, then

$$
\begin{aligned}
\psi(b-a) & =(b-a)+e_{r s}(b-a)-(b-a) e_{r s}-e_{r s}(b-a) e_{r s} \\
& =(b-a)+\left(b_{s s}-a_{s s}-b_{r r}+a_{r r}\right) e_{r s} .
\end{aligned}
$$

This means $b_{r r}-a_{r r}=b_{s s}-a_{s s}$ for all $r \neq s$, that is, $b-a$ must be central.

This implies that there exists $\gamma \in F$ such that we may write $a=b+\gamma I$. For simplicity we replace $\gamma I$ by $\gamma$.

In light of this we have that $[[a x+x b, x], a x+x b]=\left[b x^{2}-x^{2} b,(b+\gamma) x+x b\right]$. Let $i \neq j$ and choose $x=e_{i i}+e_{i j}$. By calculation it follows

$$
\left[\left[b\left(e_{i i}+e_{i j}\right)-\left(e_{i i}+e_{i j}\right) b,\left(e_{i i}+e_{i j}\right)\right],(b+\gamma)\left(e_{i i}+e_{i j}\right)+\left(e_{i i}+e_{i j}\right) b\right]=0
$$

and left multiplying by $e_{j j}$ we have

$$
e_{j j} b e_{i i}(\gamma+2 b)+e_{j j} b e_{i j}(\gamma+2 b) .
$$

Right multiplying $(A)$ by $e_{i i}$ one has

$$
b_{j i}\left(\gamma+2 b_{i i}+2 b_{j i}\right)=0 .
$$

Fix now $x=e_{i i}-e_{i j}$. By calculation we have

$$
\left[\left[b\left(e_{i i}-e_{i j}\right)-\left(e_{i i}-e_{i j}\right) b,\left(e_{i i}-e_{i j}\right)\right],(b+\gamma)\left(e_{i i}-e_{i j}\right)+\left(e_{i i}-e_{i j}\right) b\right]=0
$$

and left multiplying by $e_{j j}$ it follows

$$
e_{j j} b e_{i i}(\gamma+2 b)-e_{j j} b e_{i j}(\gamma+2 b) \text {. }
$$

Right multiplying $(B)$ by $e_{i i}$ we have

$$
b_{j i}\left(\gamma+2 b_{i i}-2 b_{j i}\right)=0 .
$$

By equations (1) and (2), and $\operatorname{since} \operatorname{char}(R) \neq 2$, we get $b_{j i}=0$, that is, $b$ is a diagonal matrix. As above we may introduce any $\psi \in \operatorname{Aut}_{F}(R)$ such that $\left[\psi(b) x^{2}-x^{2} \psi(b),(b+\gamma) x+x b\right]$ and so, by the previous cases, $\psi(b-a)$ is a diagonal matrix. In particular, for any $r \neq s$, if $\psi(x)=\left(1+e_{r s}\right) x\left(1-e_{r s}\right)$, then

$$
\begin{aligned}
\psi(b-a) & =(b-a)+e_{r s}(b-a)-(b-a) e_{r s}-e_{r s}(b-a) e_{r s} \\
& =(b-a)+\left(b_{s s}-a_{s s}-b_{r r}+a_{r r}\right) e_{r s} .
\end{aligned}
$$

This means $b_{r r}-a_{r r}=b_{s s}-a_{s s}$ for all $r \neq s$, hence $b-a$ must be central and so $a, b \in Z(R)$. 


\section{The result in prime rings}

Before beginning the proof of the main theorem, for the sake of completeness, we prefer to recall some basic notations, definitions and some easy consequences of the result of Kharchenko [11] about the differential identities on a prime ring $R$. We refer to Chapter 7 in [2] for a complete and detailed description of the theory of generalized polynomial identities involving derivations.

We denote by $Q$ the Martindale quotients ring of $R$ and let $C=Z(Q)$ be the extended centroid of $R$ ([2], Chapter 2).

It is well known that any derivation of a prime ring $R$ can be uniquely extended to a derivation of its Martindale quotients $\operatorname{ring} Q$, and so any derivation of $R$ can be defined on the whole $Q$ ([2], p. 87).

Now, we denote by $\operatorname{Der}(Q)$ the set of all derivations on $Q$. By a derivation word we mean an additive map $\Delta$ of the form $\Delta=d_{1} d_{2} \cdots d_{m}$, with each $d_{i} \in \operatorname{Der}(Q)$. Then a differential polynomial is a generalized polynomial, with coefficients in $Q$, of the form $\Phi\left(\Delta_{j}\left(x_{i}\right)\right)$ involving noncommutative indeterminates $x_{i}$ on which the derivations words $\Delta_{j}$ act as unary operations. The differential polynomial $\Phi\left(\Delta_{j}\left(x_{i}\right)\right)$ is said a differential identity on a subset $T$ of $Q$ if it vanishes for any assignment of values from $T$ to its indeterminates $x_{i}$.

Let $D_{\text {int }}$ be the $C$-subspace of $\operatorname{Der}(Q)$ consisting of all inner derivations on $Q$ and let $d$ be a non-zero derivation on $R$. By Theorem 2 in [11] we have the following result (see also Theorem 1 in [14]):

Fact 1. If $d$ is a non-zero derivation on $R$ and $\Phi\left(x_{1}, \ldots, x_{n}, d\left(x_{1}\right), \ldots, d\left(x_{n}\right)\right)$ is a differential identity on $R$, then one of the following holds:

(1) either $d \in D_{\text {int }}$;

(2) or $R$ satisfies the generalized polynomial identity

$$
\Phi\left(x_{1}, \ldots, x_{n}, y_{1}, \ldots, y_{n}\right) .
$$

Fact 2. In [13] Lee proved that every generalized derivation can be uniquely extended to a generalized derivation of $U$ and thus all generalized derivations of $R$ will be implicitly assumed to be defined on the whole $U$ and obtained the following result: (Theorem 3 in [13]) Every generalized derivation $\delta$ on a dense right ideal of $R$ can be uniquely extended to $U$ and assumes the form $\delta(x)=a x+d(x)$ for some $a \in U$ and a derivation $d$ on $U$. Hence in all that follows the generalized derivation $\delta$ will be of the form $\delta=L_{a}+d$, where $L_{a}$ denote the left multiplication by some element a.

We study now the case when $d$ is a $Q$-inner derivation:

Proposition 1. Let $R$ be a prime ring of characteristic different from $2, Z(R)$ the center of $R, d$ an inner derivation of $R$ induced by some $q \in Q$ and $\delta=$ $L_{a}+d$ a generalized derivation of $R$ for some $a \in R$. If $[[\delta(x), x], \delta(x)]=0$ for all $x \in R$, then either $R$ is commutative or $a, q \in Z(R)$, that is, $\delta(x)=a x$.

Proof. Let $d(x)=[q, x]$ for all $x \in R$. Trivially we consider the case when either $a \notin Z(R)$ or $q \notin Z(R)$, if not we are done. The assumption says that $R$ 
satisfies the non-trivial generalized polynomial identity

$$
[[(a+q) x-x q, x],(a+q) x-x q] .
$$

By a theorem due to Beidar (Theorem 2 in [1]) this generalized polynomial identity is also satisfied by $Q$. In case $C$ is infinite, we have $[[(a+q) x-$ $x q, x],(a+q) x-x q]=0$ for all $x \in Q \bigotimes_{C} \bar{C}$, where $\bar{C}$ is the algebraic closure of $C$. Since both $Q$ and $Q \bigotimes_{C} \bar{C}$ are centrally closed ([5], Theorems 2.5 and 3.5], we may replace $R$ by $Q$ or $Q \otimes_{C} \bar{C}$ according as $C$ is finite or infinite. Thus we may assume that $R$ is centrally closed over $C$ which is either finite or algebraically closed and $[[(a+q) x-x q, x],(a+q) x-x q]=0$ for all $x \in R$. By Martindale's theorem [15], $R$ is a primitive ring having a non-zero socle with $C$ as the associated division ring. In light of Jacobson's theorem ([9], p. 75) $R$ is isomorphic to a dense ring of linear transformations on some vector space $V$ over $C$.

Assume first that $V$ is finite-dimensional over $C$. Then the density of $R$ on $V$ implies that $R \cong M_{k}(C)$, the ring of all $k \times k$ matrices over $C$. Since $R$ is not commutative we assume $k \geq 2$. In this case the conclusion follows by Lemma 1.

Assume next that $V$ is infinite-dimensional over $C$. Since $q \notin C$, it doesn't centralize the non-zero ideal $H=\operatorname{soc}(R)$ of $R$. Thus there exists $h_{1} \in H$ such that either $\left[a, h_{1}\right] \neq 0$ or $\left[q, h_{1}\right] \neq 0$. Moreover, because of the infinite dimensionality, $H$ does not satisfy the polynomial $\left[x_{1}, x_{2}\right]$, that is, there exist $h_{2}, h_{3} \in H$ such that $\left[h_{2}, h_{3}\right] \neq 0$. By Litoff's theorem in [6] there exists $e^{2}=e \in H$ such that $a h_{1}, h_{1} a, q h_{1}, h_{1} q, h_{2}, h_{3} \in e R e$, moreover $e R e$ is a central simple algebra finite dimensional over its center, which satisfies the generalized identity $[[(e a e+e q e) x-x e q e, x],(e a e+e q e) x-x e q e]$. Since $\left[h_{2}, h_{3}\right] \neq 0$, then $e R e$ is not commutative and so $e R e \cong M_{t}(C)$ for $t \geq 2$. By the above finite dimensional case, we have that eae, eqe $\in Z(e R e)$ which contradicts with the choice of $h_{1}$ in $e R e$.

Now we are ready to prove our main result in prime rings:

Theorem 1. Let $R$ be a prime ring of characteristic different from $2, Z(R)$ the center of $R, d$ a derivation of $R$ and $\delta=L_{a}+d$ a generalized derivation of $R$ for some $a \in R$. If $[[\delta(x), x], \delta(x)]=0$ for all $x \in R$, then either $R$ is commutative or $d=0$ and there exists $a \in C$ such that $\delta(x)=$ ax for all $x \in R$.

Proof. Assume $R$ is not commutative. If $d$ is an inner derivation, then we end up from the previous proposition. Assume that $d$ is an outer derivation of $R$.

By the assumption $R$ satisfies the differential identity $[[a x+d(x), x], a x+$ $d(x)]$. By Kharchenko's theorem [11] $R$ satisfies the polynomial identity [[ax+ $y, x], a x+y]$ and in particular $R$ satisfies the blended component $[[a x, x], a x]$, that is, [[ar, $r], a r]=0$ for all $r \in R$. As a consequence of Lemma 1 we get $a \in C$. Hence $R$ satisfies the polynomial identity [[y, $x], a x+y]$. In this case there exists $M_{k}(F)$, the ring of all matrices over a suitable field $F$, such 
that $R$ and $M_{k}(F)$ satisfy the same polynomial identities (see [8], Theorem 2 p.54 and Lemma 1 p.89). If $k=1$ then we have the contradiction that $R$ is commutative. In case $k \geq 2$, we choose $x=e_{12}, y=e_{21}$ and get the contradiction $-2 a e_{12}+2 e_{21}=0$.

\section{The results in Banach algebras}

Here $R$ will denote a complex Banach algebra. Let us introduce some well known and elementary definition for a sake of completeness.

By a Banach algebra we shall mean a complex normed algebra $R$ whose underlying vector space is a Banach space. $\operatorname{By} \operatorname{rad}(R)$ we denote the Jacobson radical of $R$. Without loss of generality we assume $R$ to be unital. In fact any Banach algebra $R$ without a unity can be embedded into a unital Banach algebra $R_{I}=R \oplus \mathbb{C}$ as an ideal of codimension one. In particular we may identity $R$ with the ideal $\{(x, 0): x \in R\}$ in $R_{I}$ via the isometric isomorphism $x \rightarrow(x, 0)$.

Our first result in this section is about continuous generalized derivations on Banach algebras:

Theorem 2. Let $R$ be a non-commutative Banach algebra, $\delta=L_{a}+d a$ continuous generalized derivations of $R$ for some $a \in R$ and some derivation $d$ of $R$. If $[[\delta(x), x], \delta(x)] \in \operatorname{rad}(R)$ for all $x \in R$, then $[a, R] \subseteq \operatorname{rad}(R)$ and $d(R) \subseteq \operatorname{rad}(R)$.

Proof. Under the assumption that $\delta$ is continuous, and since it is well known that the left multiplication map $L_{a}$ is also continuous, we have that the derivation $d$ is continuous. In [20] Sinclair proved that any continuous derivation of a Banach algebra leaves the primitive ideals invariant. Therefore, for any primitive ideal $P$ of $R$, it follows that $\delta(P) \subseteq a P+d(P) \subseteq P$, that is, also the continuous generalized derivation $\delta$ leaves the primitive ideals invariant. Denote $\frac{R}{P}=\bar{R}$ for any primitive ideals $P$. Hence we may introduce the generalized derivation $\delta_{P}: \bar{R} \rightarrow \bar{R}$ by $\delta_{P}(\bar{x})=\delta_{P}(x+P)=\delta(x)+P=a x+d(x)+P$ for all $x \in R$ and $\bar{x}=x+P$. Moreover by $[[\delta(x), x], \delta(x)] \in \operatorname{rad}(R)$ for all $x \in R$, it follows $\left[\left[\delta_{P}(\bar{x}), \bar{x}\right], \delta_{P}(\bar{x})\right]=\overline{0}$ for all $\bar{x} \in \bar{R}$. Since $\bar{R}$ is primitive, a fortiori it is prime. Thus by Theorem 1 we get that either $\bar{R}$ is commutative, that is, $[R, R] \subseteq P$, or $d=\overline{0}$ and $\bar{a} \in Z(\bar{R})$, that is, $d(R) \subseteq P$ and $[a, R] \subseteq P$.

Now let $P$ be a primitive ideal such that $\bar{R}$ is commutative. Singer and Wermer in [21] proved that any continuous linear derivation on a commutative Banach algebra maps the algebra into its radical. Moreover by a result of Johnson and Sinclair [10] any linear derivation on a semisimple Banach algebra is continuous. Hence there are no non-zero linear continuous derivations on commutative semisimple Banach algebras. Therefore $d=\overline{0}$ in $\bar{R}$, and since $[R, R] \subseteq P$ follows by the commutativity of $\bar{R}$, we also have $[a, R] \subseteq P$.

Hence in any case $d(R) \subseteq P$ and $[a, R] \subseteq P$ for all primitive ideal $P$ of $R$. Since the radical $\operatorname{rad}(R)$ of $R$ is the intersection of all primitive ideals, we get the required conclusion. 
In the special case when $R$ is a semisimple Banach algebra we may prove that:

Theorem 3. Let $R$ be a non-commutative semisimple Banach algebra, $\delta=$ $L_{a}+d$ a generalized derivations of $R$ for some $a \in R$ and some derivation $d$ of $R$. If $[[\delta(x), x], \delta(x)]=0$ for all $x \in R$, then $\delta(x)=\alpha x$ for some $\alpha \in Z(R)$.

Proof. We may prove the result in the same way of Theorem 2 and we omit the proof for brevity. Just let us to remark that at the beginning of the proof one has to use the fact that the derivation $d$ is continuous in a semisimple Banach algebra (see [20]). Hence, since the left multiplication map $L_{a}$ is continuous, also $\delta=L_{a}+d$ is continuous. Finally we use that fact that $\operatorname{rad}(R)=0$, since $R$ is semisimple.

The last result of this paper has the same flavor of Theorem 2. Now we replace the assumption concerning the continuity of the generalized derivation $\delta$ by the one that $\delta$ is spectrally bounded. Here we denote by $G(R)$ the set of invertible elements in $R$. The spectrum of an element $x$ is the subset given by $\sigma(x)=\{\lambda \in \mathbb{C}: x-\lambda e \notin G(R)\}$, where $e$ denotes the unity of $R$. The spectral radius $r(x)$ of an element $x$ is defined as $r(x)=\sup \{|\lambda|: \lambda \in \sigma(x)\}$, provided that $\sigma(x)$ is not empty. Finally, a linear map $f: R \rightarrow R$ is called spectrally bounded if there exists a constant $\alpha \geq 0$ such that $r(f(x)) \leq \alpha r(x)$ for all $x \in R$. In order to prove our final theorem we will use some results concerning spectrally bounded derivations and generalized derivations contained in [3], more precisely we need the following:

Fact 3. Every spectrally bounded derivation on a unital Banach algebra maps the algebra into the radical (Theorem 2.5 in [3]).

Fact 4. Every spectrally bounded generalized derivation leaves each primitive ideals invariant (Lemma 2.7 in [3]).

Fact 5. Let $\delta=L_{a}+d$ a generalized derivation on a unital Banach algebra $R$, for $L_{a}$ the left multiplication (by the element a) map and $d$ some derivation of $R$. Then $\delta$ is spectrally bounded if and only if both $L_{a}$ and $d$ are spectrally bounded (Theorem 2.8 in [3]).

Now we may prove the following:

Theorem 4. Let $R$ be a non-commutative Banach algebra, $\delta=L_{a}+d$ a spectrally bounded generalized derivations of $R$ for some $a \in R$ and some derivation $d$ of $R$. If $[[\delta(x), x], \delta(x)] \in \operatorname{rad}(R)$ for all $x \in R$, then $[a, R] \subseteq \operatorname{rad}(R)$ and $d(R) \subseteq \operatorname{rad}(R)$.

Proof. Write $\delta=L_{a}+d$. Since $\delta$ is spectrally bounded, by Fact 5 both $L_{a}$ and $d$ are spectrally bounded. Combining this with Fact 3 we have that $d(R) \subseteq$ $\operatorname{rad}(R)$. Moreover, since by Fact $4 \delta$ leaves each primitive ideals invariant, it follows that for any primitive ideal $P$ of $R$ we may introduce the generalized derivation $\delta_{P}: \bar{R} \rightarrow \bar{R}$ by $\delta_{P}(\bar{x})=\delta_{P}(x+P)=\delta_{P}(x)+P=a x+d(x)+P=$ 
$a x+P$ for all $x \in R$ and $\bar{x}=x+P$. As above, by $[[\delta(x), x], \delta(x)] \in \operatorname{rad}(R)$ for all $x \in R$, it follows $\left[\left[\delta_{P}(\bar{x}), \bar{x}\right], \delta_{P}(\bar{x})\right]=\overline{0}$ for all $\bar{x} \in \bar{R}$. By theorem 1 one has that either $\bar{R}$ is commutative or $[a, R] \subseteq P$.

Of course the case when $\bar{R}$ is commutative implies that $[R, R] \subseteq P$, hence $[a, R] \subseteq P$.

Hence in any case $[a, R] \subseteq P$ for all primitive ideal $P$ of $R$ and, since the radical $\operatorname{rad}(R)$ of $R$ is the intersection of all primitive ideals, we get the required conclusion.

\section{References}

[1] K. I. Beidar, Rings with generalized identities, Moscow Univ. Math. Bull. 33 (1978), no. 4, 53-58.

[2] K. I. Beidar, W. S. Martindale III, and V. Mikhalev, Rings with Generalized Identities, Monographs and Textbooks in Pure and Applied Mathematics, 196. Marcel Dekker, Inc., New York, 1996.

[3] M. Brešar and M. Mathieu, Derivations mapping into the radical. III, J. Funct. Anal. 133 (1995), no. 1, 21-29.

[4] C. L. Chuang, GPIs having coefficients in Utumi quotient rings, Proc. Amer. Math. Soc. 103 (1988), no. 3, 723-728.

[5] T. S. Erickson, W. S. Martindale III, and J. M. Osborn, Prime nonassociative algebras, Pacific J. Math. 60 (1975), no. 1, 49-63.

[6] C. Faith and Y. Utumi, On a new proof of Litoff's theorem, Acta Math. Acad. Sci. Hungar 14 (1963), 369-371.

[7] B. Hvala, Generalized derivations in rings, Comm. Algebra 26 (1998), no. 4, 1147-1166.

[8] N. Jacobson, PI-algebras, Lecture Notes in Mathematics, Vol. 441. Springer-Verlag, Berlin-New York, 1975.

[9] Structure of Rings, American Mathematical Society Colloquium Publications, Vol. 37. Revised edition American Mathematical Society, Providence, R.I. 1964.

[10] B. E. Johnson and A. M. Sinclair, Continuity of derivations and a problem of Kaplansky, Amer. J. Math. 90 (1968), 1067-1073.

[11] V. K. Kharčhenko, Differential identities of prime rings, Algebra and Logic 17 (1978), no. 2, 155-168

[12] B.-D. Kim, Derivations of semiprime rings and noncommutative Banach algebras, Commun. Korean Math. Soc. 17 (2002), no. 4, 607-618.

[13] T. K. Lee, Generalized derivations of left faithful rings, Comm. Algebra 27 (1999), no. 8, 4057-4073.

[14] _ Semiprime rings with differential identities, Bull. Inst. Math. Acad. Sinica 20 (1992), no. 1, 27-38

[15] W. S. Martindale III, Prime rings satisfying a generalized polynomial identity, J. Algebra 12 (1969), 576-584.

[16] M. Mathieu and G. J. Murphy, Derivations mapping into the radical, Arch. Math. (Basel) 57 (1991), no. 5, 469-474.

[17] M. Mathieu and V. Runde, Derivations mapping into the radical. II, Bull. London Math. Soc. 24 (1992), no. 5, 485-487.

[18] K.-H. Park, On derivations in noncommutative semiprime rings and Banach algebras, Bull. Korean Math. Soc. 42 (2005), no. 4, 671-678.

[19] E. C. Posner, Derivations in prime rings, Proc. Amer. Math. Soc. 8 (1957), 1093-1100.

[20] A. M. Sinclair, Continuous derivations on Banach algebras, Proc. Amer. Math. Soc. 20 (1969), 166-170. 
[21] I. M. Singer and J. Werner, Derivations on commutative normed algebras, Math. Ann. 129 (1955), 260-264.

[22] M. P. Thomas, The image of a derivation is contained in the radical, Ann. of Math. (2) 128 (1988), no. 3, 435-460.

[23] J. Vukman, A result concerning derivations in noncommutative Banach algebras, Glas. Mat. Ser. III 26(46) (1991), no. 1-2, 83-88.

FACULTY OF ENGINEERING

UNIVERSITY OF MESSINA

Contrada Di Dio (S.Agata)

98166, Messina, ItALy

E-mail address: defilippis@unime.it 